Article

\title{
The Interaction between Mitochondrial Oxidative Stress and Gut Microbiota in the Cardiometabolic Consequences in Diet-Induced Obese Rats
}

\author{
Adriana Ortega-Hernández 1,2, Ernesto Martínez-Martínez ${ }^{2,3}$, Ruben Gómez-Gordo ${ }^{1,2}$, \\ Natalia López-Andrés ${ }^{4}$, Amaya Fernández-Celis ${ }^{4}$, Beatriz Gutiérrrez-Miranda ${ }^{5}$, \\ María Luisa Nieto ${ }^{2,5}{ }^{(D)}$, Teresa Alarcón ${ }^{6}$, Claudio Alba ${ }^{7}$, Dulcenombre Gómez-Garre ${ }^{1,2, *(D)}$ \\ and Victoria Cachofeiro $2,3, *$
}

1 Vascular Biology and Microbiota Laboratory, Hospital Clínico San Carlos-Instituto de Investigación Sanitaria San Carlos (IdISSC), 28040-Madrid, Spain; a.ortega.hernandez@hotmail.com (A.O.-H.); ruben.gomezgordo@gmail.com (R.G.-G.)

2 Ciber de Enfermedades Cardiovasculares (CIBERCV), Instituto de Salud Carlos III, 28029-Madrid, Spain; ernmarti@ucm.es (E.M.-M.); ml.nieto@csic.es (M.L.N.)

3 Departamento de Fisiología, Facultad de Medicina, Universidad Complutense de Madrid and Instituto de Investigación Sanitaria Gregorio Marañón (IiSGM), 28007 Madrid, Spain

4 Cardiovascular Translational Research, Navarrabiomed, Complejo Hospitalario de Navarra (CHN), Universidad Pública de Navarra (UPNA), IdiSNA, 31008 Pamplona, Spain; natalia.lopez.andres@navarra.es (N.L.-A.); amaya.fernandez.decelis@navarra.es (A.F.-C.)

5 Instituto de Biología y Genética Molecular, CSIC-Universidad de Valladolid, 47003 Valladolid, Spain; bgutimiranda@gmail.com

6 Servicio de Microbiología, Hospital Universitario de La Princesa, Instituto de Investigación Sanitaria La Princesa, Departamento de Medicina Preventiva, Salud Pública y Microbiología, Facultad de Medicina, Universidad Autónoma de Madrid, 28006 Madrid, Spain; talarcon@helicobacterspain.com

7 Sección Departamental de Farmacia Galénica y Tecnología Alimentaria, Facultad de Veterinaria, Universidad Complutense de Madrid, 28040-Madrid, Spain; claudioalbarubio@gmail.com

* Correspondence: mgomezgarre@salud.madrid.org (D.G.-G.); vcara@ucm.es (V.C.); Tel.: +34-91-330-3000 or +34-91-330-7769 (D.G.-G.); +34-91-394-1489 (V.C.); Fax: +34-91-394-1628 (V.C.)

Received: 11 June 2020; Accepted: 18 July 2020; Published: 21 July 2020 updates

\begin{abstract}
Background: The objective of this study is to determine the role of mitochondrial oxidative stress in the dysbiosis associated with a high fat diet in rats. In addition, the impact of gut microbiota (GM) in the cardiometabolic consequences of diet-induced obesity in rats has been evaluated. Methods: Male Wistar rats were fed either a high fat diet (HFD) or a control (CT) one for 6 weeks. At the third week, one-half of the animals of each group were treated with the mitochondrial antioxidant MitoTempo (MT; $0.7 \mathrm{mgKg}^{-1} \mathrm{day}^{-1}$ i.p). Results: Animals fed an HFD showed a lower microbiota evenness and diversity in comparison to CT rats. This dysbiosis is characterized by a decrease in Firmicutes/Bacteroidetes ratio and relevant changes at family and genera compared with the CT group. This was accompanied by a reduction in colonic mucin-secreting goblet cells. These changes were reversed by MT treatment. The abundance of certain genera could also be relevant in the metabolic consequences of obesity, as well as in the occurrence of cardiac fibrosis associated with obesity. Conclusions: These results support an interaction between GM and mitochondrial oxidative stress and its relation with development of cardiac fibrosis, suggesting new approaches in the management of obesity-related cardiometabolic consequences.
\end{abstract}

Keywords: cardiac fibrosis; insulin resistance; microbiota; mucins; obesity 


\section{Introduction}

Nutrition is a key modulator of oxidative stress in the human body. Even under physiological conditions, nutrient intake is accompanied by a postprandial oxidative stress, with mitochondria being the major source of reactive oxygen species (ROS) [1]. Mitochondrial ROS production plays an important role in cell signaling and homeostasis. However, an excessive oxidative stress production could cause mitochondrial dysfunction and trigger cell damage and death by affecting its structures [2-4]. Clinical and experimental studies have demonstrated that mitochondrial dysfunction can participate in the deleterious consequences of several pathological conditions, including obesity [5-7]. In this sense, we have previously reported that mitochondrial ROS participate in the development of myocardial fibrosis associated with obesity, which can lead to diastolic dysfunction and could consequently promote heart failure [6,8]. Obesity is also associated with alterations in glucose homeostasis, in which mitochondrial ROS can also participate and that can further facilitate the cardiovascular complications in the context of obesity $[9,10]$.

The gastrointestinal (GI) system contains a complex and active population of microorganisms known as the gut microbiota (GM), which can contribute to a variety of host processes, from energetic metabolism to modulation of the immune system [11,12]. The mechanisms regulating microbiota composition and diversity are multifactorial, with diet being a major driver [12,13]. Numerous studies have demonstrated a link between microbiota and obesity, as well as between insulin resistance and diabetes [14,15]. Obese subjects show reduced richness and diversity of GM, with an alteration in the specific pattern of the main phyla found in humans and rodents, Firmicutes and Bacteroidetes [16,17].

Different data have shown that GM can promote storage of calories as fat, thereby influencing the development and maintenance of obesity through different mechanisms, which includes the production of bacterial metabolites $[11,16]$. In addition, alterations in intestinal barrier increase the permeability to bacterial metabolites that can reach the circulation and induce the synthesis of proinflammatory cytokines that influence the function of distal organs $[18,19]$. It is well-known that obesity is associated with a low-grade inflammatory stage.

A crosstalk between mitochondria and GM has been suggested. Mitochondria and microbiota not only share many structural and functional activities, but mitochondria can also affect the microbiome diversity and microbiota can affect mitochondrial function. This interaction can occur at different levels and mechanisms, including oxidative stress, and may be crucial for human health [20,21]. Several studies have reported that mitochondria ROS modulate the gut epithelial barrier, thereby influencing microbiot ROS [20]. By contrast, metabolites produced by GM modulate mitochondrial energy metabolism and activities [20,22,23]. Interestingly, diet could be an important modulator of mitochondria function, since the production of metabolites by microbiota is dependent on dietary compounds [12,13]. Therefore, the main purpose of this study was to evaluate whether mitochondrial oxidative stress can affect cardiometabolic consequences of diet-induced obesity through the modulation of the GM composition. To address this aim, we evaluated the impact of a high fat diet (HFD) on fecal microbiota composition and whether this effect could be modified by the administration of a mitochondrial targeted antioxidant. In addition, the interactions between GM and cardiac fibrosis and insulin resistance observed in obese rats were evaluated.

\section{Methods}

This study was performed following the Animal Care and Use Committee of Universidad Complutense of Madrid and Dirección General de Medio Ambiente, Comunidad de Madrid, which approved all experimental procedures according to the Spanish Policy for Animal Protection RD53/2013, which meets the European Union Directive 2010/63/UE (PROEX 242/15). 


\subsection{MitoTempo Administration}

The mitochondrial targeted antioxidant MitoTempo (MT) was obtained from Merck Sigma Aldrich (St. Louis, MO, USA). MT treatment was administered i.p once a day at the dose of $0.7 \mathrm{mg} / \mathrm{Kg}$ from the third week on. The MT dose was chosen based on a previous publication [8].

\subsection{Animals and Experimental Groups}

Male Wistar rats of $150 \mathrm{~g}$, purchased from Envigo (Barcelona, Spain), were fed either a HFD (HFD, $35 \%$ fat; Envigo Teklad \#TD.03307, Haslett, MI, USA; $n=16)$ or a standard diet (CT, $3.5 \%$ fat; Envigo Teklad \#TD.2014; $n=16$ ) for 6 weeks. Half of the animals of each group received the mitochondrial antioxidant MT. Therefore, 4 experimental groups were included in the study: CT ( $n=8$ animals), MT ( $n=8$ animals), HFD ( $n=8$ animals) and HFD + MT ( $n=8$ animals). All animals were held in a light- and temperature-controlled room with free access to diet and tap water. Food, water intake and weight were periodically controlled throughout the experimental period. At the end of the study, fasted animals were euthanized, and blood, white adipose tissue pads, heart, colon and fecal content were collected. For each animal, adiposity index was calculated as the sum of white fat pads/[(body weight-fat pad weight) $\times$ 100] [24].

\subsection{Blood Biochemistry}

Plasma glucose concentration was determined using an automatic analyzer (Vitros 5600, OrthoClinical). Plasma insulin levels were measured using a specific quantitative sandwich enzyme immunoassay (Mercodia, Uppsala, Sweden) according to the manufacturer's instructions, and peripheral insulin sensitivity was calculated based on the Homeostasis Model Assessment (HOMA) [25].

\subsection{Western Blotting}

Total proteins were prepared as previously described [6] from cardiac homogenates from obese and control rats. Proteins were separated by SDS-PAGE on $12.5 \%$ polyacrylamide gels and transferred to polyvinylidene difluoride membranes (Hybond-P; Amersham Biosciences, Piscataway, NJ, USA). Membranes were probed with primary antibodies for $\alpha$-smooth muscle actin ( $\alpha$-SMA; dilution: 1:500; Merck Sigma Aldrich), fibronectin (dilution: 1:500; Merck Millipore, Darmstad, Germany), periostin (dilution: 1:1000; Santa Cruz Biotechnology, Dallas, TX, USA) and vimentin (dilution 1:1000, Santa Cruz Biotechnology). Stain free detection was used for loading control (Bio-Rad Laboratories, Hercules, CA, USA). Results are expressed as an $\mathrm{n}$-fold increase over the values of the control group in densitometric arbitrary units.

\subsection{Mucin Level Analysis in Goblet Cells}

Colon tissue samples were dehydrated, embedded in paraffin and cut into $5 \mu \mathrm{m}$-thick sections. Mucin levels in goblet cells were quantified in Alcian Blue (AB)/periodic acid-Schiff (PAS)-stained sections using ImageJ image analysis program. Acidic mucins stain blue with AB and neutral mucins stain pink with PAS, while mixtures of neutral and acidic mucins appear purple. For each sample, 5 to 7 fields with at least ten complete contiguous colon crypts were analyzed with a 20X objective under transmitted light microscope. The area occupied by mucins was identified as the ratio of percentage of mucin content to the total tissue area. A single researcher unaware of the experimental groups performed the analysis.

\subsection{Isolation of Microbial DNA}

Microbial DNA was extracted from $150 \mathrm{mg}$ of the fecal content from each animal using the QIAamp DNA Stool kit (Qiagen, Hilden, Germany) according to the manufacturer's instructions. DNA integrity was evaluated with an Agilent 2100 Bioanalyzer System and DNA concentration was 
determined with a Qubit 3.0 Fluorometer using the dsDNA HS (High Sensitivity) Assay (Thermo Fisher Scientific, Madrid, Spain).

\subsection{Amplification and Sequencing of the $16 \mathrm{~S}$ rDNA Gene}

For each sample, bacterial $16 \mathrm{~S}$ rDNA was amplified by polymerase chain reaction (PCR) using the Ion $16 \mathrm{~S}^{\mathrm{TM}}$ Metagenomics Kit (Thermo Fisher Scientific) that uses two primer pools to amplify seven hypervariable regions (V2, V4, V8 and V3, V6-7, V9, respectively). The amplification protocol was as follows: $95^{\circ} \mathrm{C}$ for $10 \mathrm{~min}$ followed by 25 cycles of $95^{\circ} \mathrm{C}$ for $30 \mathrm{~s}, 58^{\circ} \mathrm{C}$ for $30 \mathrm{~s}$ and $72{ }^{\circ} \mathrm{C}$ for $20 \mathrm{~s}$, and a final step of $7 \mathrm{~min}$ at $72{ }^{\circ} \mathrm{C}$. Equal volumes of the two primer reactions were pooled and PCR amplicons purified with paramagnetic beads technology (CleanPCR, Labclinics, Barcelona, Spain). Barcoded libraries were prepared from 5 ng of DNA per sample using the Ion Plus Fragment Library Kit (Thermo Fisher Scientific) to end-repair amplicons and the Ion Xpress ${ }^{\mathrm{TM}}$ Barcode Adapters Kit (Thermo Fisher Scientific) to ligate the barcode adapters, according to the manufacturer's instructions. Libraries were diluted to $22 \mathrm{pM}$ prior to clonal amplification by emulsion PCR with the Ion OneTouch ${ }^{\mathrm{TM}}$ 2 System using the Ion $520^{\mathrm{TM}}$ and Ion $530^{\mathrm{TM}}$ Kit-OT2 (Thermo Fisher Scientific), and then sequenced on an Ion S5 System using a Ion 520 ${ }^{\mathrm{TM}}$ Chip (Thermo Fisher Scientific).

\subsection{Bioinformatic Analysis}

Base calling, low quality filtering, removal of polyclonal reads and demultiplexing were automated by Torrent Suite ${ }^{\mathrm{TM}}$ software (v5.10.0; Thermo Fisher Scientific). Primer regions were removed and sequences were trimmed to $150 \mathrm{bp}$ using self-developed Python scripts. FASTQ files were analyzed using QIIME 2 software (v2020.2) to dereplicate reads and remove singletons. SILVA 16S rRNA gene database (v132) was used to perform a reference-based clustering of operational taxonomic units (OTUs) at 99\% similarity. OTUs with fewer than 10 observations across all samples were discarded, and relative OTU abundances were computed as percent proportions based on the total number of reads per sample.

Alpha and beta-diversity analyses were performed from the filtered OTU table to avoid any biases caused by the presence of rare OTUs using self-developed Python and R scripts. To assess alpha diversity, four metrics were calculated: observed OTUs, Chao1 richness estimate, Shannon diversity index and Pielou's evenness index.

To perform beta diversity analysis, Bray-Curtis dissimilarity and Jensen-Shannon divergence (JSD) distance matrices were computed. Principal coordinate analysis (PCoA) was applied to these metrics and the compositional dissimilarity between groups and samples was visualized in two-dimensional PCoA plots.

Taxonomic assignment of OTUs was carried out using QIIME 2 and SILVA 16 S taxonomy. Picrust2 software (v2.3.0-b) was used to predict the functional profiles of microbial communities in terms of Kyoto Encyclopedia of Genes and Genomes (KEGG) functional orthologs and metabolic pathways. Linear discriminant analysis (LDA) effect size (LEfSe) analysis was performed to differentially identify abundant taxa and functional features among groups. Taxa with a relative abundance less than $0.01 \%$ were filtered for this analysis.

\subsection{Statistical Analysis}

Continuous variables are expressed as mean \pm standard error of the mean (SEM). Normality of distributions was verified by means of the Kolmogorov-Smirnov test. Data normally distributed were analyzed using a one-way analysis of variance, followed by a Newman-Keuls to assess specific differences among groups or conditions. The differences in alpha and beta diversity metrics among groups were assessed using the non-parametric Kruskal-Wallis test, and the permutation-based multivariate analysis of variance (PERMANOVA) with 999 permutations, respectively. LEfSe analysis consists of the application of a Kruskal-Wallis test, followed by an LDA step, which assigns to each feature an LDA score that assesses its association with the categorical variables of interest. A threshold 
of $\alpha=0.05$ was considered statistically significant, establishing an LDA score cut-off of 3.0 for taxa and 2.0 for functional features. Association between genera relative abundances and cardiac collagen and HOMA index was studied using Pearson's correlations. Genera showing $|\mathrm{r}|<0.6$ in their correlation with all the clinical parameters were filtered out. Multivariate analysis, considering either cardiac fibrosis or HOMA as the dependent variable, was performed with a linear regression model by means of a backward stepwise method. In consecutive steps, variables that were statistically significant in the univariable analysis were included in the linear regression model. Data analysis was performed using Python scripts and the statistical program SPSS v25.0 (SPSS Inc, Chicago, IL, USA).

\section{Results}

\subsection{Effects of MitoTempo in Metabolic and Cardiac Parameters of Diet-Induced Obese Rats}

The administration of the mitochondrial antioxidant MT to HFD rats reduced the increase in adiposity, and in the following metabolic parameters: fasting glucose, insulin levels and, in consequence, HOMA index showed by HFD-induced obese rats (Table 1). In addition, HFD animals receiving MT showed a significant decrease in relative heart weight and cardiac interstitial fibrosis [8], but also $\alpha$-SMA, fibronectin and periostin protein expression (Figure 1) as compared with HFD rats treated with vehicle. MT did not affect any of these parameters in CT animals (Table 1 and Figure 1A-D).

Table 1. Effect of the mitochondrial antioxidant MitoTempo (MT; $0.7 \mathrm{mg} / \mathrm{Kg} /$ day i.p) on metabolic parameters in rats fed a normal chow (CT) and high fat diet (HFD) fed rats.

\begin{tabular}{ccccc}
\hline Variable & CT & MT & HFD & HFD + MT \\
\hline Adiposity index $(\%)$ & $3.49 \pm 0.26$ & $3.52 \pm 0.22$ & $8.39 \pm 0.41^{* * *}$ & $6.9 \pm 0.55^{*,+++}$ \\
\hline Glucose $(\mathrm{mg} / \mathrm{dL})$ & $98.8 \pm 4.6$ & $95.2 \pm 2.8$ & $114.6 \pm 2.1^{* *}$ & $104.3 \pm 2.5^{+}$ \\
\hline Insulin $(\mathrm{pg} / \mathrm{mL})$ & $72.2 \pm 7.7$ & $60.9 \pm 5.9$ & $241.6 \pm 30.8^{* * *}$ & $132.7 \pm 16.5^{*,+++}$ \\
\hline HOMA index & $2.50 \pm 0.33$ & $1.98 \pm 0.19$ & $9.28 \pm 1.1^{* * *}$ & $4.8 \pm 0.7^{*}+\mathrm{t++}$ \\
\hline
\end{tabular}

Data are mean \pm SEM, $n=7-8$ in each group. ${ }^{*} p<0.05 ;{ }^{* *} p<0.01 ;{ }^{* * *} p<0.001$ vs. CT group ${ }^{+} p<0.05 ;{ }^{+t+} p$ vs. HFD group.

A

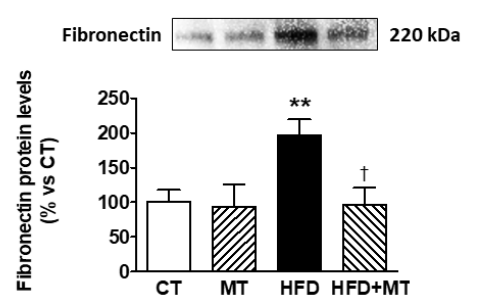

c

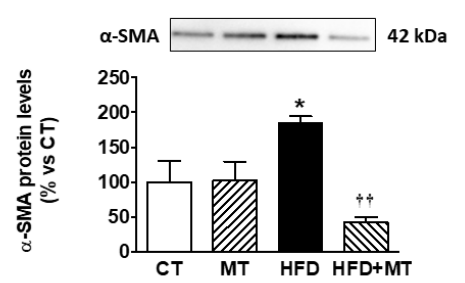

B

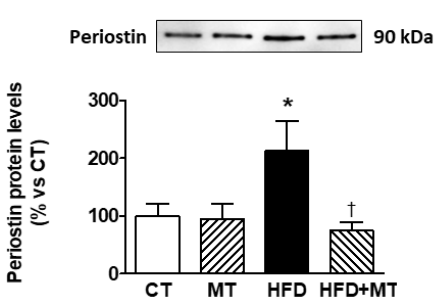

D

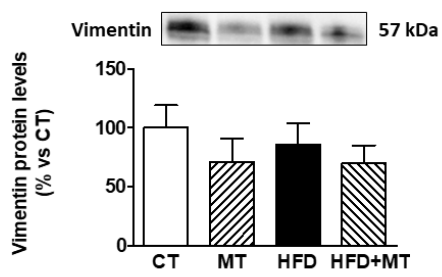

Figure 1. Protein levels of (A) fibronectin, (B) periostin, (C) $\alpha$-smooth muscle actin (SMA) and (D) vimentin in heart from control rats fed a normal chow (CT) and rats fed a high fat diet (HFD) treated with vehicle or with the mitochondrial antioxidant MitoTempo (MT; $0.7 \mathrm{mg} / \mathrm{Kg} /$ day i.p). Bars graphs represent the mean \pm SEM of 6-8 animals. Protein densitometry was expressed in arbitrary units (AU) once normalized to stain-free gel for protein. ${ }^{*} p<0.05 ;{ }^{* *} p<0.01$ vs. CT group. ${ }^{\dagger} p<0.05,{ }^{+\dagger} p<0.01$ vs. HFD group. 


\subsection{Effects of MitoTempo in the Mucin Levels in the Colon of Diet-Induced Obese Rats}

We used the combined analysis of AB/PAS staining to evaluate both neutral and acidic mucin secreting cells in colon simultaneously. As shown in Figure 2, there is a predominance of goblet cells containing acidic mucins over neutral mucins in the rat colon, in a proportion $\sim 3: 1$, in $\mathrm{CT}$ rats fed a standard diet. HFD rats showed a significant drop-off in the overall AB/PAS staining when compared with CT animals that was reversed by MT (Figure 2A,B). Since MT neither affected the levels of colonic goblet cells containing neutral and acidic granules in CT animals (Figure 2) nor metabolic parameters (Table 1) or cardiac proteins (Figure 1), to simplify, only data from CT, HFD or HFD+MT groups will be presented from now on.

A
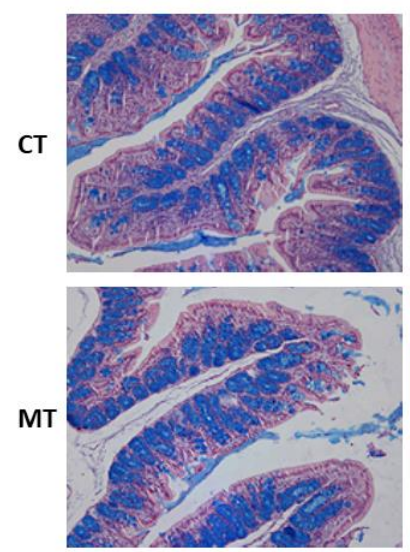

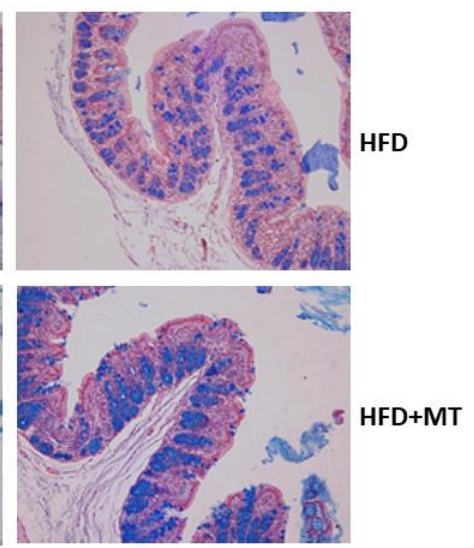

B

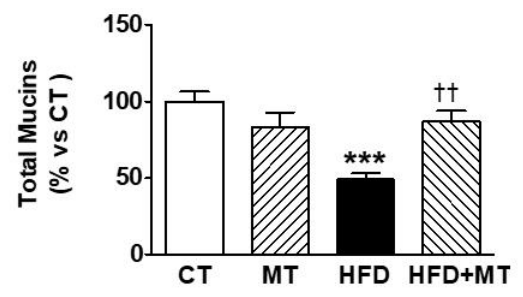

Figure 2. (A) Representative microphotographs and (B) quantification of total mucin levels in colon from control animals fed a normal chow (CT) and animals fed a high fat diet (HFD) treated with vehicle or with the mitochondrial antioxidant MitoTempo (MT; $0.7 \mathrm{mg} / \mathrm{Kg} /$ day i.p) stained with Alcian Blue (AB)/periodic acid-Schiff (PAS) examined by light microscopy (magnification $20 \times$ ). Bar graphs represent the mean \pm SEM of $5-6$ animals normalized to for CT group. ${ }^{* * *} p<0.001$ vs. CT group; ${ }^{++} p<0.01$ vs. HFD group.

\subsection{Effects of MitoTempo in Gut Microbiota Diversity of Diet-Induced Obese Rats}

Fecal DNA was isolated from 8 CT rats, 8 HFD rats and 7 HFD rats treated with MT. After 16S rDNA sequencing, 6,332,097 reads were successfully classified into 6119 OTUs that were used for downstream analysis.

First, we studied the alpha diversity of GM, which refers to the intrinsic biodiversity of the samples, in CT, HFD and HFD+MT rats. No differences were found in richness among any group, as assessed by observed OTUs and Chao1 indexes (Table 2). By contrast, we observed that Shannon and Pielou's indexes were significantly reduced in HFD rats, thus revealing lower microbiota evenness and overall diversity in comparison to control rats. The administration of MT was able to reverse these changes (Table 2).

Next, we estimated beta diversity of GM in CT, HFD and HDF+MT rats. Bray-Curtis dissimilarity and JSD indexes revealed significant differences between HFD group as compared with CT group. MT partially, but significantly, restored GM composition, although it was not normalized since significant differences were still observed between MT-treated animals and CT ones (Table 2). 
Table 2. Alpha and beta diversity indexes in rats fed a normal chow (CT) and high fat diet (HFD) and treated with the antioxidant mitochondrial MitoTempo (MT; $0.7 \mathrm{mg} / \mathrm{Kg} /$ day i.p).

\begin{tabular}{cccc}
\hline Variable & CT & HFD & HFD + MT \\
\hline Observed OTUs & $3267 \pm 121$ & $3495 \pm 117$ & $3458 \pm 98$ \\
Chao1 richness index & $3945 \pm 100$ & $4168 \pm 1128$ & $4095 \pm 61$ \\
Shannon index & $7.93 \pm 0.14$ & $7.51 \pm 0.10^{*}$ & $7.95 \pm 0.14^{\dagger}$ \\
Pielou's evenness index & $0.68 \pm 0.011$ & $0.64 \pm 0.01^{*}$ & $0.68 \pm 0.013^{\dagger}$ \\
Bray-Curtis dissimilarity & $0.549 \pm 0.017$ & $0.423 \pm 0.02^{* *}$ & $0.62 \pm 0.041^{*}+$ \\
JSD distance & $0.483 \pm 0.013$ & $0.388 \pm 0.015^{* *}$ & $0.521 \pm 0.033^{*}+$
\end{tabular}

Jensen-Shannon divergence (JSD). Data are mean \pm SEM, $n=7-8$ in each group. ${ }^{*} p<0.05$ vs. CT group; ${ }^{* *} p<0.01$ vs. CT group; ${ }^{\dagger} p<0.05$ vs. HFD group.

\subsection{Effects of MitoTempo in Gut Microbiota Composition of HFD-Induced Obese Rats}

Taxonomic assignment of OTUs gave rise to the identification of 15 phyla, 25 classes, 42 orders, 79 families and 275 genera in the GM. In all groups, the most abundant phyla were Firmicutes, Bacteroidetes, Proteobacteria and Tenericutes, although with significant differences in their abundances among groups (Figure 3A-D). GM composition in HFD rats was more abundant in Bacteroidetes and Proteobacteria, and less in Firmicutes and Tenericutes when compared to CT animals. The administration of MT to HFD rats normalized the abundance of bacteria belonging to phyla Firmicutes, Bacteroidetes and Proteobacteria. However, it did not affect the reduction in Tenericutes observed in HFD rats (Figure 3A-D).

A

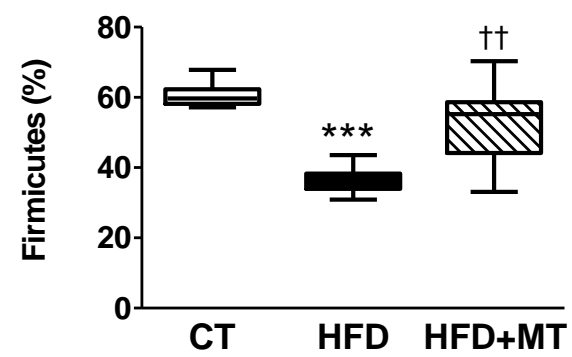

C

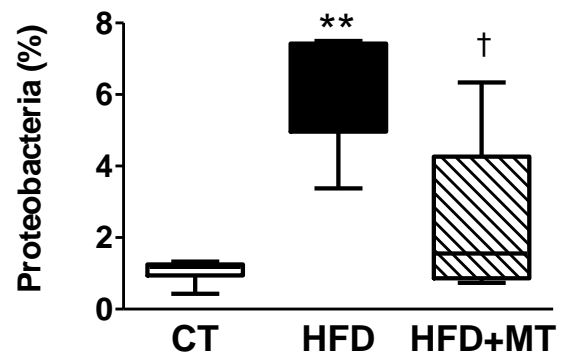

B

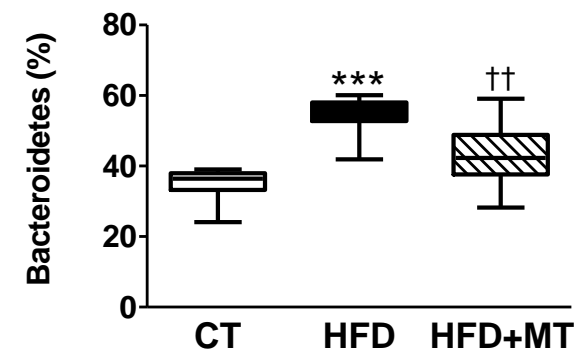

D

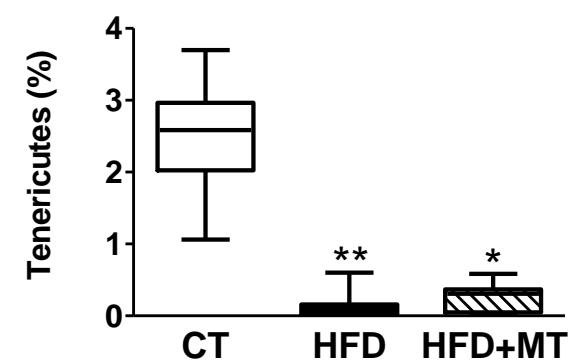

Figure 3. Boxplot showing total relative abundance of reads of the four most abundant taxa at Phylum level in the gut microbiota (A) Firmicutes, (B) Bacteroidetes, (C) Protobacteria and (D) Tenericutes in feces from control animals fed a normal chow (CT) and animals fed a high fat diet (HFD) treated with vehicle or with the mitochondrial antioxidant MitoTempo (MT; $0.7 \mathrm{mg} / \mathrm{Kg} /$ day i.p). Upper, middle and lower lines represent first quartiles, medians and third quartiles. The whiskers represent a $1.5 *$ inter-quartile range. Data are expressed as percentage of total reads. ${ }^{*} p<0.05 ;{ }^{* *} p<0.01$; ${ }^{* * *} p<0.001$ vs. CT group. ${ }^{\dagger} p<0.05,{ }^{+\dagger} p<0.01$ vs. HFD group. 
LEfSe analysis of bacterial taxa constituting GM, at the family level, is shown in Figure 4. We found that 6 families characterized the CT group: 3 belonged to the Firmicutes phylum (Lachnospiraceae, Ruminococcaceae and Lactobacillaceae); 1 to the Bacteroidetes phylum (Muribaculaceae), 1 to the Proteobacteria phylum (Caldicoprobacteraceae) and 1 to the Patescibacteria phylum (Saccharimonadaceae) (Figure 4A). Obese animals showed a decrease in all these families with respect to CT (Figure 4B). By contrast, obesity was accompanied by an enrichment of 14 families with respect to CT group (Figure 4B). Consistently with phylum composition: 4 families belonged to the Bacteroidetes phylum (Bacteroidaceae, Rikenellaceae, Barnesiellaceae and Tannerellaceae); 4 to the Proteobacteria phylum (Desulfovibrionaceae, Enterobacteriaceae, Pasteurellaceae and Nitrosomonadaceae), 3 to the Firmicutes phylum (Peptostreptococcaceae, Christensenellaceae and Erysipelotrichaceae), 2 to the Actinobacteria phylum (Coriobacteriaceae, Eggerthellaceae) and 1 to the Verrucomicrobia phylum (Akkermansiaceae). Ten of these families characterized the HFD group: Bacteroidaceae and Rikenellaceae, and Desulfovibrionaceae, Enterobacteriaceae, Pasteurellaceae and Nitrosomonadaceae from Bacteroidetes and Proteobacteria phyla, respectively, and Erysipelotrichaceae and Coriobacteriaceae, and Eggerthellaceae from Firmicutes and Actinobacteria phyla, respectively (Figure 4A). MT treatment was able to reverse the abundance of some families: it increased the diminution of Lachnospiraceae, Ruminococcaceae and Muribaculaceae observed in HFD rats, and decreased the rise in the number of families of Bacteroidetes (Bacteroidaceae, Rikenellaceae, Barnesiellaceae and Tannerellaceae), and Verrucomicrobia phylum (Akkermansiaceae), and in some families of Proteobacteria (Enterobacteriaceae and Pasteurellaceae) (Figure 4C). Two families belonging to Firmicutes phylum characterized the group of HFD animals treated with MT: Peptostreptococcaceae and Bacillaceae.

A

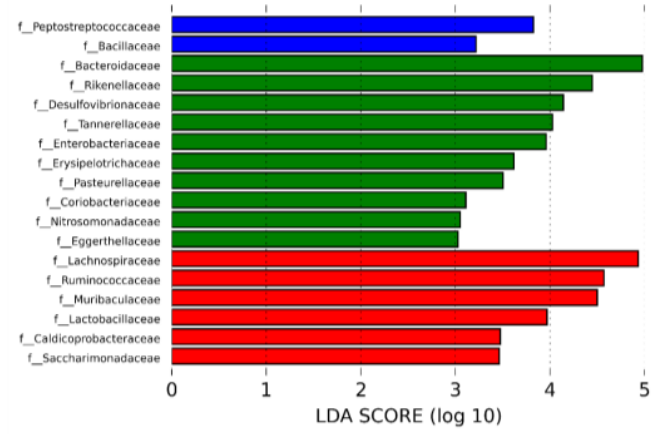

C

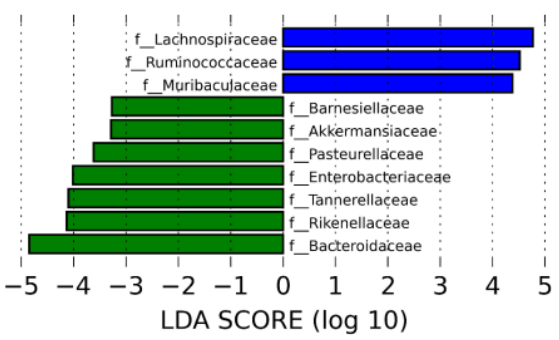

B

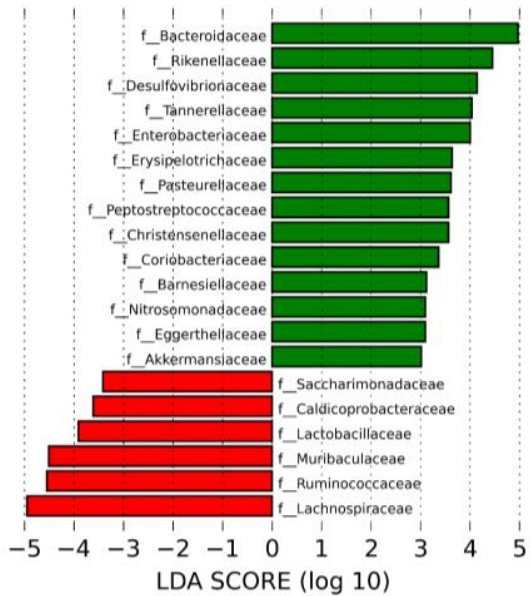

$\square$ CT $\square$ HFD $\square$ HFD+MT

Figure 4. LEfSe analysis showing taxonomic differential abundance at family level in feces from control animals fed a normal chow (CT) and animals fed a high fat diet (HFD) treated with vehicle or with the mitochondrial antioxidant MitoTempo (MT; $0.7 \mathrm{mg} / \mathrm{Kg} /$ day i.p). (A) Significantly different families among CT, HFD and HFD+MT groups. (B) Significantly enriched and depleted families between CT and HFD groups. (C) Significantly enriched and depleted families between HFD and HFD + MT groups. The length of the horizontal bars represents the LDA score (effect size). $p<0.05$; LDA score $>$ 3.0.

At the genus level, GM of CT and HFD rats showed 14 and 17 genera differentially found, respectively, while obese animals treated with MT were characterized by 7 genera (Figure 5A). Regarding genera driving divergences between groups, 30 genera were enriched in HFD animals with respect to CT animals: 16 belonged to the Firmicutes phylum, 4 to the Bacteroidetes phylum 
(Bacteroides presented the highest difference), 8 to the Proteobacteria phylum and 1 to the Verrucomicrobia and Actinobacteria phyla (Figure 5B). By contrast, 19 genera were reduced in HFD animals as compared with controls, 14 belonging to Firmicutes phylum (Ruminococcus 1 presented the highest difference), 2 to Proteobacteria and 1 to Bacteroidetes and Saccharibacteria (Figure 5B). MT treatment was able to reverse only some changes of the GM composition of obese animals. It increased the abundance of 7 genera out of 19 (6 genera belonging to Firmicutes phylum, with Acetitomaculum presenting the highest difference) and a single genus belonging to Actinobacteria (Pygmaiobacter). It reduced the content of 15 genera out of 30 ( 8 belonging to Proteobacteria phylum, 3 belonging to Bacteroidetes, with Bacteroides presenting the highest difference, 3 to Firmicutes and a single genus to Verrucomicrobia phylum (Figure 5C)).

A

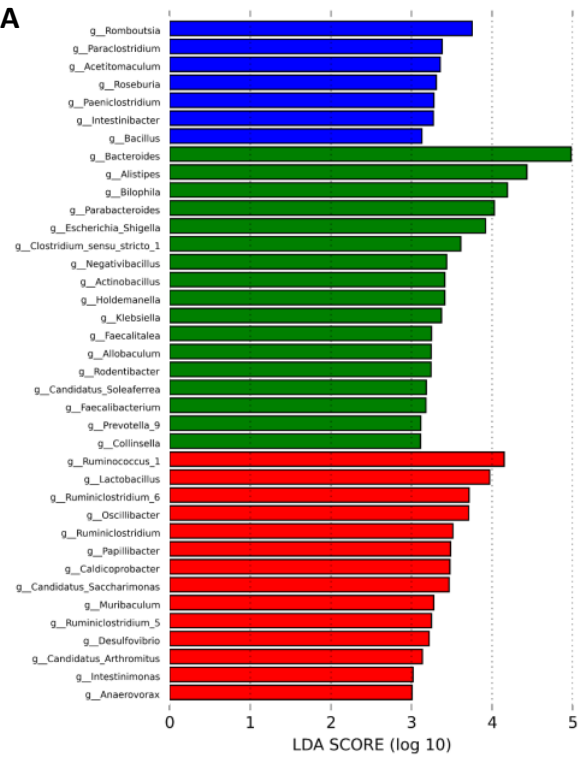

B

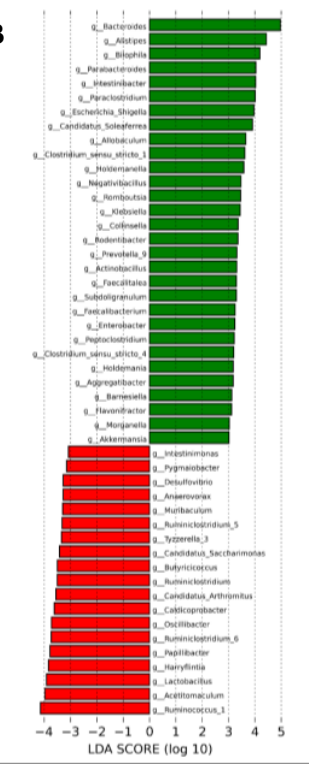

C

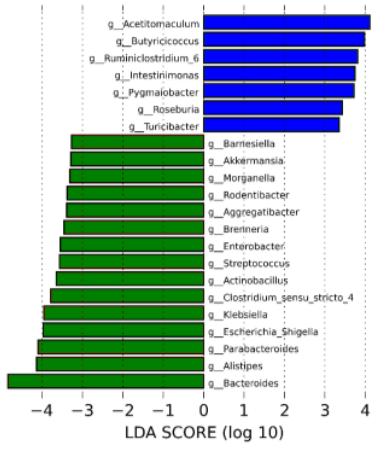

$\square \mathrm{CT}$ $\square$ HFD

HFD+MT

Figure 5. LEfSe analysis showing taxonomic differential abundance at genus level in feces from control animals fed a normal chow (CT) and animals fed a high fat diet (HFD) treated with vehicle or with the mitochondrial antioxidant MitoTempo (MT; $0.7 \mathrm{mg} / \mathrm{Kg} /$ day i.p). (A) Significantly different genera among CT, HFD and HFD+MT groups. (B) Significantly enriched and depleted genera between CT and HFD groups. (C) Significantly enriched and depleted genera between HFD and HFD + MT groups. The length of the horizontal bars represents the LDA score (effect size). $p<0.05$; LDA score $>3.0$.

\subsection{Relationship between Genera Abundance and Metabolic and Cardiac Parameters}

Relative abundances of all identified genera were correlated with cardiac collagen levels, HOMA and adiposity indexes. Among genera displaying a significant correlation with these parameters, Morganella showed the highest correlation with cardiac collagen (Pearson's $\mathrm{r}=0.831$ ); Candidatus soleaferrea with HOMA (Pearson's $r=0.791$ ) and Muribaculum with adiposity (Pearson's $r=-0.761$ ). Multivariate analysis showed that 1 genus from Proteobacteria phylum (Morganella) and 4 from Firmicutes phylum (Eubacterium oxidoreducens group, Lachnospiraceae ND3007 group, Erysipelotrichaceae UCG-003, Ruminococcus 1 and Streptococcus) were independent predictors of collagen content (Table 3).

One genus from Bacteroidetes (uncultured Muribaculaceae) and 4 from Firmicutes (Candidatus soleaferrea, Peptoclostridium, Eubacterium oxidoreducens group and Holdemania were independent predictors of HOMA index (Table 3). 
Table 3. Multivariate models for collagen content and Homeostasis Model Assessment (HOMA) index.

\begin{tabular}{|c|c|c|c|c|}
\hline Genus & Collagen Conten & & HOMA Index & \\
\hline- & B (95\% CI) & $p$ & B $(95 \%$ CI $)$ & $p$ \\
\hline Eubacterium oxidoreducens group & $-0.73(-1.17$ to -0.28$)$ & 0.003 & $-2.36(-3.73$ to -0.98$)$ & 0.002 \\
\hline Streptococcus & $6.26(0.30$ to 12.22$)$ & 0.041 & & \\
\hline Lachnospiraceae ND3007 group & $-3.42(-6.17$ to -0.67$)$ & 0.018 & & \\
\hline Erysipelotrichaceae UCG-003 & $-63.64(-109.45$ to -17.08$)$ & 0.01 & & \\
\hline Ruminococcus 1 & $-0.13(-0.25$ to -0.11$)$ & 0.034 & & \\
\hline Morganella & 10.71 (6.91 to 14.51$)$ & $<0.0001$ & & \\
\hline Muribaculaceae (uncultured bacterium) & & & $-0.457(-0.667$ to -0.246$)$ & $<0.0001$ \\
\hline Candidatus Soleaferrea & & & 253.77 (125.49 to 382.05$)$ & 0.001 \\
\hline Holdmania & & & $-107.70(-177.80$ to -37.70$)$ & 0.005 \\
\hline Peptoclostridium & & & 82.47 (5.56 to 159.38$)$ & 0.037 \\
\hline
\end{tabular}

\subsection{Effects of MitoTempo in Gut microbiota Metabolism of Diet-Induced Obese Rats}

To identify metabolic pathways significantly different among rat groups, a LEfSe analysis was performed. Obesity was accompanied by a reduction in the pathways related to butanoate (ko00650) and propanoate (ko00640) metabolism, which were improved in those animals treated with MT (Figure 6A,B). By contrast, an increase in the abundance of genera involved in glutathione metabolism (ko00480) was observed in HFD-induced obese animals as compared with control ones, which was normalized by treatment with the mitochondrial antioxidant (Figure 6C). An increase in abundant taxa of genera involved in lipopolysaccharide (LPS) production (ko00540) was found in HFD as compared with $\mathrm{CT}$, and MT was able to normalize it (Figure 6D).

A

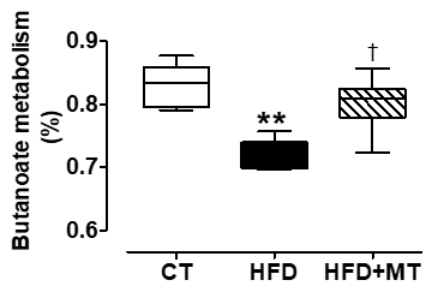

C

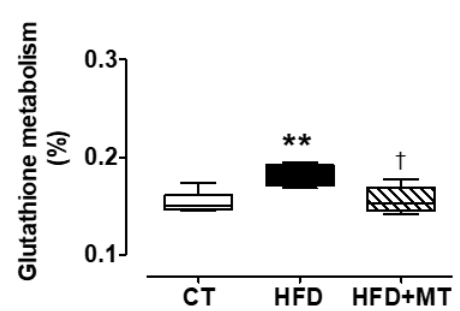

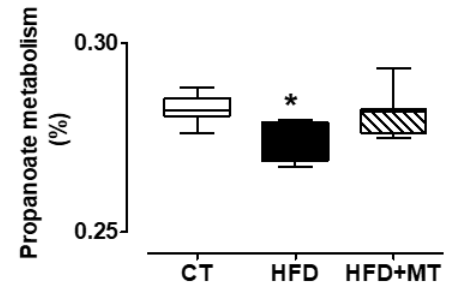

D

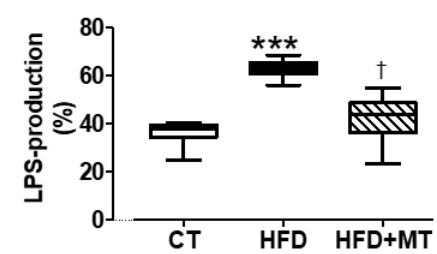

Figure 6. Boxplot showing total relative abundance of metabolic pathways related to gut microbiota in (A) butanoate metabolism, (B) propanoate metabolism and (C) glutathione metabolism and (D) percentage of bacteria involved in lipopolysaccharyde (LPS) production in feces from control animals fed a normal chow (CT) and animals fed a high fat diet (HFD) treated with vehicle or with the mitochondrial antioxidant MitoTempo (MT; $0.7 \mathrm{mg} / \mathrm{Kg} /$ day i.p). Upper, middle and lower lines represent first quartiles, medians and third quartiles. The whiskers represent a $1.5 *$ inter-quartile range. Data are expressed as percentage of total reads. ${ }^{*} p<0.05 ;{ }^{* *} p<0.01 ; * * *<0.001$ vs. CT group. ${ }^{+} p<0.05$ vs. HFD group. 


\section{Discussion}

Obesity is closely associated with GM dysbiosis. We have previously shown the relevance of mitochondrial oxidative stress in cardiac alterations associated with obesity [8]. In this study, we have now demonstrated that the administration of the mitochondrial antioxidant MT prevents HFD diet-induced obesity, insulin resistance and cardiac fibrosis in association with a preservation of mucingoblet cells in colon and a restoration of GM composition. Interestingly, we observed that changes in the abundance of certain genera could be relevant not only in the development of obesity and its metabolic consequences but also in the occurrence of cardiac fibrosis associated with obesity.

As already reported, HFD rats show a different gut environment with respect to lean animals characterized by low diversity and richness of gut microbiome, a feature associated with obesity and its metabolic alterations [26,27]. As in humans, the two dominant phyla observed in both lean and obese rats are Firmicutes and Bacteroidetes, although with significant differences about their relative distributions among the studies [28]. This variety in the results may rely, at least in part, on different methodological approaches (metagenomics sequencing, sample microbial load) but also in the area of the gut from which the samples were taken [16,29]. In this study, HFD rats showed a low Firmicutes/Bacteroidetes ratio with elevation of the Bacteroidetes phylum and decreasing levels of the Firmicutes phylum. Bacteria belonging to Firmicutes are important producers of short-chain fatty acids (SCFAs) and have been associated with healthy intestinal barrier and a reduction in endotoxin leakage [30-32]. Bacteroidetes, the largest phylum of Gram-negative, can release LPS involved in inflammatory activation, mainly when the intestinal mucosal barrier is disrupted. In this sense, obese animals showed a reduction in mucin-secreting goblet cells that may result in a defective mucin production, pointing to a damaged intestinal barrier.

GM dysbiosis has been associated with insulin resistance, a common alteration observed in the context of obesity $[14,33]$. Our data show that 5 genera were independent predictors of HOMA (an index of insulin resistance), 2 of them in a positive manner (Candidatus soleaferrea and Peptoclostridium) and 3 in a negative one (uncultured Muribaculaceae, Eubacterium oxidoreducens group and Holdemania). Different data have also reported the association between insulin resistance and the levels of these genera [14,34-36].

Fibrosis is a well-known feature of the cardiac remodeling associated with obesity that can favor functional alterations by reducing cardiac relaxing capability and therefore increase its filling pressure. Our data show that the fibrosis observed in obese animals not only involved an increase in collagen content [8], the main component of extracellular matrix (ECM), but also of other components of ECM such as periostin and fibronectin. Of special interest, periostin is a potent modulator of cell-matrix interaction that has been implicated in the crosstalk between multiple signaling pathways including TGF- $\beta$ [37]. In line with our results, treatment of diabetic animals with the antioxidant resveratrol resulted in a significant reduction in myofibroblast activation, and inhibited the expression of periostin via TGF- $\beta$ signaling [38]. Concerning fibronectin, its blockade has been described as attenuating cardiac fibrosis in an experimental model of heart failure [39]. This excessive ECM deposit was associated with an increase in myofibroblasts as indicated by the increased $\alpha$-SMA levels, the cell mainly responsible for fibrosis. The analysis show that 6 genera were independent predictors of fibrosis content, both in positive (Morganella and Streptococcus) and in a negative manner (Eubacterium oxidoreducens group, Lachnospiraceae ND3007 group, Erysipelotrichaceae UCG-003 and Ruminococcus 1). In a model of cirrhosis in rats, the treatment with antibiotics induced an exacerbation of hepatic fibrosis that was associated with overgrowth by Morganella morganii [40]. Similarly, Streptococcus can trigger the progression of pulmonary fibrosis in two different mouse models of pulmonary fibrosis [41], supporting a profibrotic capacity of these genera. No information regarding the potential role in fibrosis has been reported about the genera negatively associated with cardiac fibrosis in our study. However, they are more abundant in healthy subjects than in subjects with different pathological conditions [42,43].

Several mechanisms could account for the beneficial effects induced by the mitochondrial anti-oxidant. The impact of this microbiota profile on the gut's mucosal integrity and the translocation 
of microbial cell wall components, like LPS from Gram-negative bacteria and other toxic metabolites, could be the potential mechanisms involved in the impact of the gut dysbiosis in the cardiometabolic consequences in the context of obesity $[19,40,42-44]$. Dysbiosis can affect the integrity of the gut barrier, due to abnormal or reduced numbers of mucous cell production, resulting in diminished mucus production and both enhanced permeability and transit of antigens [45].

One of the hallmarks of obesity and obesity-related pathologies is the occurrence of chronic low-grade inflammation that may affect the fibrotic development [46]. This inflammation-related process can be initiated by LPS [47]. Our metabolic pathway analysis showed an increase in LPS-producing bacteria in the gut of obese rats that was inhibited by the anti-oxidant. Moreover, MT-treated obese rats also restored bacteria genera involved in the pathways of propanoate and butanoate metabolism, SCFA essential for the maintenance of intestinal epithelium physiology by regulating the cellular turnover and barrier functions. In addition, SCFA modulate the inflammatory response by suppressing the production of pro-inflammatory mediators induced by LPS or inducing differentiation of regulatory T cells $[29,48,49]$.

Moreover, obese animals show an increase in bacteria genera involved in the pathways of glutathione metabolism that protects against oxidative stress, especially in the heart. Disturbances in cardiac glutathione homeostasis has been associated with cardiac lipotoxicity in diet-induced obesity in mice [50]. The fact that these alterations were reversed by MT further support a crosstalk between microbiota-mitochondria.

In conclusion, our data demonstrated that the administration of a mitochondrial anti-oxidant to HFD obese rats modulates the GM composition, promoting a change in ratio Firmicutes to Bacteroidetes, thereby decreasing bacteria genera associated with insulin resistance, fibrosis and inflammation. These results further support an interaction between GM and mitochondrial oxidative stress, suggesting new approaches in the management of obesity-related cardiometabolic consequences.

Author Contributions: Conceptualization, E.M.-M., D.G.-G., M.L.N. and V.C.; methodology, A.O.-H., A.F.-C., B.G.-M., E.M.-M., D.G.-G. and V.C.; formal analysis, A.O.-H., R.G.-G., T.A., C.A., D.G.-G., M.L.N. and V.C.; data curation, A.O.-H., E.M.-M., N-L.-A., D.G.-G. and V.C.; writing, E.M.-M., R.G.-G., N.L.-A., M.L.N., D.G.-G. and V.C.; review and editing, A.O.-H., E.M.-M., R.G.-G., T.A., C.A., A.F.-C., N.L.-A., B.G.-M., M.L.N., D.G.-G., M.L.N. and V.C.; funding acquisition, E.M.-M., M.L.N. and V.C. All authors have read and agreed to the published version of the manuscript.

Funding: This work was supported by Instituto de Salud Carlos III-Fondo Europeo de Desarrollo Regional (FEDER) (PI18/00257; CIBERCV) a way to build Europe, Ministerio de Economía y Competitividad (SAF2016-81063). EMM was supported by a contract from CAM (Atracción de talento). BG was funded by the FPI Program from the Government of Castilla y León (co-funded by FSE).

Acknowledgments: We thank Raquel Jurado-López for their technical help. We thank Anthony DeMarco for his help in editing.

Conflicts of Interest: The authors declare no conflicts of interest.

\section{References}

1. Diamanti-Kandarakis, E.; Papalou, O.; Kandaraki, E.A.; Kassi, G. Mechanisms in Endocrinology: Nutrition as a mediator of oxidative stress in metabolic and reproductive disorders in women. Eur. J. Endocrinol. 2017, 176, R79-R99. [CrossRef] [PubMed]

2. Green, D.R.; Galluzzi, L.; Kroemer, G. Mitochondria and the autophagy-inflammation-cell death axis in organismal aging. Science 2011, 333, 1109-1112. [CrossRef]

3. Redza-Dutordoir, M.; Averill-Bates, D.A. Activation of apoptosis signalling pathways by reactive oxygen species. Biochim. Biophys. Acta 2016, 1863, 2977-2992. [CrossRef] [PubMed]

4. Wei, H.; Cong, X. The effect of reactive oxygen species on cardiomyocyte differentiation of pluripotent stem cells. Free Radic. Res. 2018, 52, 150-158. [CrossRef]

5. de Mello, A.H.; Costa, A.B.; Engel, J.D.G.; Rezin, G.T. Mitochondrial dysfunction in obesity. Life Sci. 2018, 192, 26-32. [CrossRef] 
6. Jimenez-Gonzalez, S.; Marin-Royo, G.; Jurado-Lopez, R.; Bartolome, M.V.; Romero-Miranda, A.; Luaces, M.; Islas, F.; Nieto, M.L.; Martinez-Martinez, E.; Cachofeiro, V. The Crosstalk between Cardiac Lipotoxicity and Mitochondrial Oxidative Stress in the Cardiac Alterations in Diet-Induced Obesity in Rats. Cells 2020, 9, 451. [CrossRef]

7. Marin-Royo, G.; Rodriguez, C.; Le Pape, A.; Jurado-Lopez, R.; Luaces, M.; Antequera, A.; Martinez-Gonzalez, J.; Souza-Neto, F.V.; Nieto, M.L.; Martinez-Martinez, E.; et al. The role of mitochondrial oxidative stress in the metabolic alterations in diet-induced obesity in rats. FASEB J. Off. Publ. Fed. Am. Soc. Exp. Biol. 2019, 33, 12060-12072. [CrossRef]

8. Gutierrez-Tenorio, J.; Marin-Royo, G.; Martinez-Martinez, E.; Martin, R.; Miana, M.; Lopez-Andres, N.; Jurado-Lopez, R.; Gallardo, I.; Luaces, M.; San Roman, J.A.; et al. The role of oxidative stress in the crosstalk between leptin and mineralocorticoid receptor in the cardiac fibrosis associated with obesity. Sci. Rep. 2017, 7, 16802. [CrossRef] [PubMed]

9. Ortega, F.B.; Lavie, C.J.; Blair, S.N. Obesity and Cardiovascular Disease. Circ. Res. 2016, 118, $1752-1770$. [CrossRef]

10. Isomaa, B.; Almgren, P.; Tuomi, T.; Forsen, B.; Lahti, K.; Nissen, M.; Taskinen, M.R.; Groop, L. Cardiovascular morbidity and mortality associated with the metabolic syndrome. Diabetes Care 2001, 24, 683-689. [CrossRef] [PubMed]

11. Miyamoto, J.; Igarashi, M.; Watanabe, K.; Karaki, S.I.; Mukouyama, H.; Kishino, S.; Li, X.; Ichimura, A.; Irie, J.; Sugimoto, Y.; et al. Gut microbiota confers host resistance to obesity by metabolizing dietary polyunsaturated fatty acids. Nat. Commun. 2019, 10, 4007. [CrossRef]

12. Maslowski, K.M.; Mackay, C.R. Diet, gut microbiota and immune responses. Nat. Immunol. 2011, 12, 5-9. [CrossRef]

13. Flint, H.J.; Duncan, S.H.; Scott, K.P.; Louis, P. Links between diet, gut microbiota composition and gut metabolism. Proc. Nutr. Soc. 2015, 74, 13-22. [CrossRef]

14. Wang, S.; Huang, M.; You, X.; Zhao, J.; Chen, L.; Wang, L.; Luo, Y.; Chen, Y. Gut microbiota mediates the anti-obesity effect of calorie restriction in mice. Sci. Rep. 2018, 8, 13037. [CrossRef]

15. Khan, M.J.; Gerasimidis, K.; Edwards, C.A.; Shaikh, M.G. Role of Gut Microbiota in the Aetiology of Obesity: Proposed Mechanisms and Review of the Literature. J. Obes. 2016, 2016, 7353642. [CrossRef]

16. Angelakis, E.; Armougom, F.; Million, M.; Raoult, D. The relationship between gut microbiota and weight gain in humans. Future Microbiol. 2012, 7, 91-109. [CrossRef]

17. Duncan, S.H.; Lobley, G.E.; Holtrop, G.; Ince, J.; Johnstone, A.M.; Louis, P.; Flint, H.J. Human colonic microbiota associated with diet, obesity and weight loss. Int. J. Obes. 2008, 32, 1720-1724. [CrossRef]

18. Kabouridis, P.S.; Lasrado, R.; McCallum, S.; Chng, S.H.; Snippert, H.J.; Clevers, H.; Pettersson, S.; Pachnis, V. The gut microbiota keeps enteric glial cells on the move; prospective roles of the gut epithelium and immune system. Gut Microbes 2015, 6, 398-403. [CrossRef]

19. Natividad, J.M.; Verdu, E.F. Modulation of intestinal barrier by intestinal microbiota: Pathological and therapeutic implications. Pharmacol. Res. 2013, 69, 42-51. [CrossRef]

20. Saint-Georges-Chaumet, Y.; Edeas, M. Microbiota-mitochondria inter-talk: Consequence for microbiota-host interaction. Pathog. Dis. 2016, 74, ftv096. [CrossRef]

21. Clark, A.; Mach, N. The Crosstalk between the Gut Microbiota and Mitochondria during Exercise. Front. Physiol. 2017, 8, 319. [CrossRef] [PubMed]

22. Donohoe, D.R.; Garge, N.; Zhang, X.; Sun, W.; O'Connell, T.M.; Bunger, M.K.; Bultman, S.J. The microbiome and butyrate regulate energy metabolism and autophagy in the mammalian colon. Cell Metab. 2011, 13, 517-526. [CrossRef] [PubMed]

23. den Besten, G.; van Eunen, K.; Groen, A.K.; Venema, K.; Reijngoud, D.J.; Bakker, B.M. The role of short-chain fatty acids in the interplay between diet, gut microbiota, and host energy metabolism. J. Lipid Res. 2013, 54, 2325-2340. [CrossRef] [PubMed]

24. Kozak, L.P.; Newman, S.; Chao, P.M.; Mendoza, T.; Koza, R.A. The early nutritional environment of mice determines the capacity for adipose tissue expansion by modulating genes of caveolae structure. PLoS ONE 2010, 5, e11015. [CrossRef]

25. Gutch, M.; Kumar, S.; Razi, S.M.; Gupta, K.K.; Gupta, A. Assessment of insulin sensitivity/resistance. Indian J. Endocrinol. Metab. 2015, 19, 160-164. [CrossRef] 
26. Rastelli, M.; Knauf, C.; Cani, P.D. Gut Microbes and Health: A Focus on the Mechanisms Linking Microbes, Obesity, and Related Disorders. Obesity 2018, 26, 792-800. [CrossRef]

27. Vallianou, N.; Stratigou, T.; Christodoulatos, G.S.; Dalamaga, M. Understanding the Role of the Gut Microbiome and Microbial Metabolites in Obesity and Obesity-Associated Metabolic Disorders: Current Evidence and Perspectives. Curr. Obes. Rep. 2019, 8, 317-332. [CrossRef]

28. Sze, M.A.; Schloss, P.D. Looking for a Signal in the Noise: Revisiting Obesity and the Microbiome. mBio 2016, 7. [CrossRef]

29. Lee, C.J.; Sears, C.L.; Maruthur, N. Gut microbiome and its role in obesity and insulin resistance. Ann. N. Y. Acad. Sci. 2020, 1461, 37-52. [CrossRef]

30. Truax, A.D.; Chen, L.; Tam, J.W.; Cheng, N.; Guo, H.; Koblansky, A.A.; Chou, W.C.; Wilson, J.E.; Brickey, W.J.; Petrucelli, A.; et al. The Inhibitory Innate Immune Sensor NLRP12 Maintains a Threshold against Obesity by Regulating Gut Microbiota Homeostasis. Cell Host Microbe 2018, 24, 364-378.e366. [CrossRef]

31. Vujicic, M.; Saksida, T.; Despotovic, S.; Bajic, S.S.; Lalic, I.; Koprivica, I.; Gajic, D.; Golic, N.; Tolinacki, M.; Stojanovic, I. The Role of Macrophage Migration Inhibitory Factor in the Function of Intestinal Barrier. Sci. Rep. 2018, 8, 6337. [CrossRef]

32. Vital, M.; Karch, A.; Pieper, D.H. Colonic Butyrate-Producing Communities in Humans: An Overview Using Omics Data. mSystems 2017, 2. [CrossRef]

33. Do, M.H.; Lee, E.; Oh, M.J.; Kim, Y.; Park, H.Y. High-Glucose or -Fructose Diet Cause Changes of the Gut Microbiota and Metabolic Disorders in Mice without Body Weight Change. Nutrients 2018, 10, 761. [CrossRef]

34. Lippert, K.; Kedenko, L.; Antonielli, L.; Kedenko, I.; Gemeier, C.; Leitner, M.; Kautzky-Willer, A.; Paulweber, B.; Hackl, E. Gut microbiota dysbiosis associated with glucose metabolism disorders and the metabolic syndrome in older adults. Benef. Microbes 2017, 8, 545-556. [CrossRef] [PubMed]

35. Han, L.; Zhao, L.H.; Zhang, M.L.; Li, H.T.; Gao, Z.Z.; Zheng, X.J.; Wang, X.M.; Wu, H.R.; Zheng, Y.J.; Jiang, X.T.; et al. A Novel Antidiabetic Monomers Combination Alleviates Insulin Resistance Through Bacteria-Cometabolism-Inflammation Responses. Front. Microbiol. 2020, 11, 173. [CrossRef] [PubMed]

36. Walker, J.M.; Eckardt, P.; Aleman, J.O.; da Rosa, J.C.; Liang, Y.; Iizumi, T.; Etheve, S.; Blaser, M.J.; Breslow, J.L.; Holt, P.R. The effects of trans-resveratrol on insulin resistance, inflammation, and microbiota in men with the metabolic syndrome: A pilot randomized, placebo-controlled clinical trial. J. Clin. Transl. Res. 2019, 4, 122-135. [PubMed]

37. Walker, J.T.; McLeod, K.; Kim, S.; Conway, S.J.; Hamilton, D.W. Periostin as a multifunctional modulator of the wound healing response. Cell Tissue Res. 2016, 365, 453-465. [CrossRef]

38. Wu, H.; Li, G.N.; Xie, J.; Li, R.; Chen, Q.H.; Chen, J.Z.; Wei, Z.H.; Kang, L.N.; Xu, B. Resveratrol ameliorates myocardial fibrosis by inhibiting ROS/ERK/TGF-beta/periostin pathway in STZ-induced diabetic mice. BMC Cardiovasc. Disord. 2016, 16, 5. [CrossRef]

39. Valiente-Alandi, I.; Potter, S.J.; Salvador, A.M.; Schafer, A.E.; Schips, T.; Carrillo-Salinas, F.; Gibson, A.M.; Nieman, M.L.; Perkins, C.; Sargent, M.A.; et al. Inhibiting Fibronectin Attenuates Fibrosis and Improves Cardiac Function in a Model of Heart Failure. Circulation 2018, 138, 1236-1252. [CrossRef]

40. Plummer, J.L.; Ossowicz, C.J.; Whibley, C.; Ilsley, A.H.; Hall, P.D. Influence of intestinal flora on the development of fibrosis and cirrhosis in a rat model. J. Gastroenterol. Hepatol. 2000, 15, 1307-1311. [CrossRef]

41. Knippenberg, S.; Ueberberg, B.; Maus, R.; Bohling, J.; Ding, N.; Tort Tarres, M.; Hoymann, H.G.; Jonigk, D.; Izykowski, N.; Paton, J.C.; et al. Streptococcus pneumoniae triggers progression of pulmonary fibrosis through pneumolysin. Thorax 2015, 70, 636-646. [CrossRef] [PubMed]

42. Singh, H.; Torralba, M.G.; Moncera, K.J.; DiLello, L.; Petrini, J.; Nelson, K.E.; Pieper, R. Gastro-intestinal and oral microbiome signatures associated with healthy aging. GeroScience 2019, 41, 907-921. [CrossRef] [PubMed]

43. Cui, X.; Ye, L.; Li, J.; Jin, L.; Wang, W.; Li, S.; Bao, M.; Wu, S.; Li, L.; Geng, B.; et al. Metagenomic and metabolomic analyses unveil dysbiosis of gut microbiota in chronic heart failure patients. Sci. Rep. 2018, 8, 635. [CrossRef] [PubMed]

44. Kimura, I.; Ozawa, K.; Inoue, D.; Imamura, T.; Kimura, K.; Maeda, T.; Terasawa, K.; Kashihara, D.; Hirano, K.; Tani, T.; et al. The gut microbiota suppresses insulin-mediated fat accumulation via the short-chain fatty acid receptor GPR43. Nat. Commun. 2013, 4, 1829. [CrossRef] 
45. Ghosh, S.S.; Wang, J.; Yannie, P.J.; Ghosh, S. Intestinal Barrier Dysfunction, LPS Translocation, and Disease Development. J. Endocr. Soc. 2020, 4, bvz039. [CrossRef]

46. Ellulu, M.S.; Patimah, I.; Khaza'ai, H.; Rahmat, A.; Abed, Y. Obesity and inflammation: The linking mechanism and the complications. Arch. Med Sci. AMS 2017, 13, 851-863. [CrossRef]

47. Gregor, M.F.; Hotamisligil, G.S. Inflammatory mechanisms in obesity. Annu. Rev. Immunol. 2011, 29, 415-445. [CrossRef]

48. Huang, W.; Zhou, L.; Guo, H.; Xu, Y.; Xu, Y. The role of short-chain fatty acids in kidney injury induced by gut-derived inflammatory response. Metab. Clin. Exp. 2017, 68, 20-30. [CrossRef]

49. Chang, P.V.; Hao, L.; Offermanns, S.; Medzhitov, R. The microbial metabolite butyrate regulates intestinal macrophage function via histone deacetylase inhibition. Proc. Natl. Acad. Sci. USA 2014, 111, 2247-2252. [CrossRef]

50. Ghosh, S.; Sulistyoningrum, D.C.; Glier, M.B.; Verchere, C.B.; Devlin, A.M. Altered glutathione homeostasis in heart augments cardiac lipotoxicity associated with diet-induced obesity in mice. J. Biol. Chem. 2011, 286, 42483-42493. [CrossRef]

(C) 2020 by the authors. Licensee MDPI, Basel, Switzerland. This article is an open access article distributed under the terms and conditions of the Creative Commons Attribution (CC BY) license (http://creativecommons.org/licenses/by/4.0/). 